\title{
Towards the deformation quantization of linearized gravity
}

\author{
Hernando Quevedo $^{1,2, *}$ and Julio G. Tafoya ${ }^{1,+}$ \\ ${ }^{1}$ Instituto de Ciencias Nucleares \\ Universidad Nacional Autónoma de México \\ A. Postal 70-543, México D.F. 04510, MEXICO \\ ${ }^{2}$ Department of Physics \\ University of California \\ Davis, CA 95616, USA
}

\begin{abstract}
We present a first attempt to apply the approach of deformation quantization to linearized Einstein's equations. We use the analogy with Maxwell equations to derive the field equations of linearized gravity from a modified Maxwell Lagrangian which allows the construction of a Hamiltonian in the standard way. The deformation quantization procedure for free fields is applied to this Hamiltonian. As a result we obtain the complete set of quantum states and its discrete spectrum.
\end{abstract}

PACS numbers: $04.20 . \mathrm{Cv}, 04.25 .-\mathrm{g}, 11.10 .-\mathrm{Nx}$

*Electronic address: quevedo@physics.ucdavis.edu

${ }^{\dagger}$ Electronic address: antonio@nuclecu.unam.mx 


\section{INTRODUCTION}

One of the main differences between the states of a classical system and those of a quantum system consists in that the latter cannot be represented as points in phase space. In the canonical quantization approach this fact is taken into account by considering states as eigenfunctions of operators which act on Hilbert space. The eigenvalues of the operators are then interpreted as quantum observables. So, one of the main steps of the canonical quantization formalism consists basically in replacing the classical observables of the physical system by operators. Commutation relations are then imposed on the operators in order to be in agreement with Heisenberg's uncertainty relation. Although this procedure is widely used in quantum mechanics and quantum field theory, it is still far from being completely understood. In particular, one expects that in certain limit the quantum system reduces to the classical one. This is usually done by applying the correspondence principle, according to which the quantum commutator must lead to the classical Poisson bracket when Planck's constant vanishes. However, it is known that in general this limit is not well-defined and inconsistent [1].

One of the main successes of deformation quantization consists in providing the correct implementation of the correspondence principle. Indeed, deformation quantization avoids the use of operators and, instead, concentrates on the algebra of classical observables (see [2, 3, 4] for recent reviews, and [5] for an elementary review.) Instead of the usual (classical) point multiplication between observables, a star-product is introduced that takes into account the non-local character of quantum observables and reduces to the classical Poisson bracket in the appropriate limit. Whereas the classical observables build a commutative algebra with respect to the point product, the same set forms a noncommutative algebra with respect to the star-product. Thus, it is not necessary to introduce new entities (operators) instead of the classical observables. The quantum observables are represented by the same functions on phase space as the classical observables. The first applications of deformation quantization concerned non-relativistic quantum mechanics [6], but this situation has changed dramatically in the past few years. This formalism has found many uses in perturbative and nonperturbative quantum field theory [7, 8, 9, 10, 11, 12, 13], quantum gravity [14], as well as in string theory [15, 16].

In this work we present a first attempt to apply the main concepts of deformation quan- 
tization to the case of linearized gravity. Our approach consists basically in representing Einstein's linearized equations as a field theory of a metric on the background of Minkowski spacetime. This approach allows us to apply in this case the procedure of deformation quantization developed for free fields. Our approach is also appropriate for getting rid of the concern regarding the quantization of a quantity (the perturbation metric) which is first considered as infinitesimal in order for Einsteins linearization to be valid. Indeed, intuitively one expects that quantum effects become important only when the gravitational field is high enough, and the perturbation metric in the standard approach does not behave this way. In the field theoretical approach the metric is arbitrary and linearized Einsteins equations follow from a variational principle. The corresponding Lagrangian is regular and the Hamiltonian turns out to be equivalent to the Hamiltonian of an infinite sum of harmonic oscillators.

This paper is structured as follows. In Section ஹwe derive Einsteins linearized equations in the standard manner and present an alternative field theoretical approach based upon the analogy between electrodynamics and linearized gravity. In Section III we review the main aspects of deformation quantization and calculate explicitly the set of quantum states and the energy spectrum of linearized gravity. Finally, in Section [V we discuss our results.

\section{LINEARIZED GRAVITY}

In this section we first review the standard approach of linearized gravity in which the field equations are obtained by linearizing Einsteins equations. Then we present an alternative approach based on the usual variational procedure of field theory and obtain the corresponding Hamiltonian.

\section{A. Linearized Einsteins equations}

In most textbooks on general relativity, linearized gravity is considered at the level of the field equations. Indeed, in the usual approach to gravity, one starts from the Einstein-Hilbert action [18]

$$
S=\int R \sqrt{-g} d^{4} x+\alpha_{m} \int \mathcal{L}_{m} d^{4} x
$$

where $R$ is the curvature scalar associated to the metric $g_{\alpha \beta},(\alpha, \beta, \ldots=0,1,2,3)$ of spacetime, $\alpha_{m}$ is a coupling constant and $\mathcal{L}_{m}$ is the Lagrangian density that represents the matter 
contents in spacetime. The variation of (2.1) with respect to the metric yields the Einstein equations

$$
R_{\alpha \beta}-\frac{1}{2} g_{\alpha \beta} R=8 \pi T_{\alpha \beta}
$$

where the energy-momentum tensor is defined in terms of the variational derivative as

$$
T_{\alpha \beta}=-\frac{\alpha_{m}}{8 \pi} \frac{1}{\sqrt{-g}} \frac{\delta \mathcal{L}_{m}}{\delta g^{\alpha \beta}} .
$$

In the weak field approximation of linearized gravity one imposes the metric $g_{\alpha \beta}=\eta_{\alpha \beta}+$ $h_{\alpha \beta}$ such that $h_{\alpha \beta}<<\eta_{\alpha \beta}$ is an infinitesimal perturbation of the background Minkowski metric, $\eta_{\alpha \beta}$. In particular, one can choose a Cartesian-like coordinate system in which $\eta_{\alpha \beta}=\operatorname{diag}(-,+,+,+)$ and $\left|h_{\alpha \beta}\right|<<1$. If we now consider the first order approximation of the left-hand side of (2.2) with respect to $h_{\alpha \beta}$, and impose the Lorentz gauge condition

$$
\bar{h}_{, \beta}^{\alpha \beta}=0, \quad \text { with } \quad \bar{h}^{\alpha \beta}=h^{\alpha \beta}-\frac{1}{2} \eta^{\alpha \beta} h, \quad h=\eta_{\alpha \beta} h^{\alpha \beta}
$$

then Einstein's equations reduce to

$$
\bar{h}_{\alpha \beta, \gamma}^{\gamma}=-16 \pi T_{\alpha \beta}
$$

\section{B. A field theoretical approach}

An alternative approach for deriving the linearized equations (2.5), in which the particular aspects of a field theory become more plausible, consists in using the analogy between Maxwell's equations of electromagnetism and linearized Einstein's equations. To show this analogy explicitly let us consider a non-zero vector $U^{\alpha}$ and define

$$
A_{\alpha}=-\frac{1}{4} \bar{h}_{\alpha \beta} U^{\beta}, \quad \text { and } \quad J_{\alpha}=-T_{\alpha \beta} U^{\beta}
$$

Calculating the D'Alembertian $A_{\alpha, \gamma}^{\gamma}$, one can see immediately that the Maxwell equations

$$
A_{\alpha, \gamma}^{\gamma}=-4 \pi J_{\alpha}
$$

are equivalent to the linearized Einstein equations (2.5) if the components of the arbitrary vector $U^{\alpha}$ satisfy the equations

$$
\bar{h}_{\alpha \beta} U_{, \gamma}^{\beta} \gamma+2 \bar{h}_{\alpha \beta, \gamma} U^{\beta, \gamma}=0
$$


On the other hand, the Lorentz gauge condition (2.4) turns out to be equivalent to the condition $A_{, \alpha}^{\alpha}=0$ if the equation

$$
\bar{h}_{\alpha \beta} U^{\beta, \alpha}=0
$$

is satisfied. In this way we see that the linearized Einstein equations can be written in the Maxwell-like form (2.7) (in the Lorentz gauge) by introducing the additional vector $U^{\alpha}$ which is required to satisfy the conditions (2.8) and (2.9). For a given metric $\bar{h}_{\alpha \beta}$, these conditions represent a system of four second order partial differential (2.8) and one first order partial differential equation (2.9) for the four components $U^{\alpha}$. So one should guarantee the existence of solutions to this system for the Maxwell-like representation (2.7) to be valid. Obviously, the trivial vector $U^{\alpha}=$ const satisfies these requirements. In principle, more general solutions might exist, but in this work we will restrict ourselves to this special case since it is sufficient for our purposes.

On the other hand, it is well known that Maxwell equations (2.7) can be obtained by varying the Lagrangian (density)

$$
\mathcal{L}_{\text {Max }}=-\frac{1}{4} F_{\alpha \beta} F^{\alpha \beta}+4 \pi A_{\alpha} J^{\alpha}, \quad \text { where } \quad F_{\alpha \beta}=A_{\beta, \alpha}-A_{\alpha, \beta},
$$

with respect to the potential $A_{\alpha}$. Let us now try to construct the corresponding Hamiltonian formalism. The configuration variables are given by the set of components $A_{\alpha}$. For the corresponding canonically conjugate momenta we obtain $\Pi_{\alpha}=\partial \mathcal{L}_{M a x} / \partial \dot{A}_{\alpha}=F_{0}{ }^{\alpha}$, where a dot denotes the derivative with respect to the time coordinate $x^{0}$. Then $\Pi_{0}=0$ and consequently we have a singular Lagrangian from which a Hamiltonian cannot be constructed. In field theory the quantization of such Lagrangians is performed by using Dirac's method for systems with constraints (see, for instance, [20]). But in the context of deformation quantization this method is still under construction [17]. In the case of Maxwell's theory, however, an alternative approach exists [21] that consists in modifying the original Maxwell Lagrangian according to (we take $J_{\alpha}=0$ for simplicity)

$$
\mathcal{L}=-\frac{1}{4} F_{\alpha \beta} F^{\alpha \beta}-\frac{1}{2}\left(A_{\alpha}^{, \alpha}\right)^{2}
$$

The field equations are again $A_{\alpha, \beta}{ }^{\beta}=0$ and the Lorentz gauge condition $A_{\alpha}{ }^{, \alpha}=0$ has to be postulated separately. After some algebraic manipulations, the Lagrangian (2.11) can be rewritten as

$$
\mathcal{L}=-\frac{1}{2} A_{\alpha, \beta} A^{\alpha, \beta}+\frac{1}{2} \Lambda_{, \beta}^{\beta}, \quad \Lambda^{\beta}=A_{, \gamma}^{\beta} A^{\gamma}-A^{\beta} A_{, \gamma}^{\gamma}
$$


The second term can be neglected as it can be transformed after integration into a surface term that does not contribute to the field equations. But the main point about the Lagrangian (2.12) is that it is regular. In fact, it can easily be seen that $\Pi_{\alpha}=\dot{A}_{\alpha}$ and the corresponding Hamiltonian is

$$
\mathcal{H}=\frac{1}{2}\left(\Pi_{\alpha} \Pi^{\alpha}+A_{\alpha, i} A^{\alpha, i}\right)
$$

Then the canonical variables of the phase space satisfy the canonical commutation relations with respect to the Poisson bracket:

$$
\left\{A_{\alpha}, \Pi_{\beta}\right\}=\delta_{\alpha \beta}, \quad\left\{A_{\alpha}, A_{\beta}\right\}=\left\{\Pi_{\alpha}, \Pi_{\beta}\right\}=0 .
$$

We will use the Hamiltonian (2.13) in the context of deformation quantization in section III. Finally, let us mention that the Lagrangian (2.12) is invariant with respect to the transformation

$$
A_{\alpha} \rightarrow A_{\alpha}^{\prime}=A_{\alpha}+\Sigma_{, \alpha}, \quad \text { with } \quad \Sigma_{, \alpha}^{\alpha}=0
$$

This is a special gauge transformation that can be used to eliminate non-physical degrees of freedom.

The main point of this alternative approach is that we now can "forget" that $\mathcal{L}$ is an approximate Lagrangian and proceed as in standard classical field theory. That is, (2.12) can be interpreted as the Lagrangian for the metric field $h_{\alpha \beta}$ which is defined on the Minkowski spacetime with metric $\eta_{\alpha \beta}$. So we are dealing with a standard field theory in which the background Minkowski metric does not interact with the field $h_{\alpha \beta}$ that now can be completely arbitrary, i.e. it is not necessarily an infinitesimal perturbation of the background metric. In this manner we can avoid the concern mentioned in the introduction about the quantization of an infinitesimal quantity.

\section{DEFORMATION QUANTIZATION}

The classical description of the evolution of a physical system is usually represented in the phase space $\Gamma$, which is a manifold of even dimension. If a (non-degenerate) symplectic twoform $\alpha$ exists on $\Gamma$, then the phase space is called a symplectic space. Observables are real valued functions defined on the phase space: $f, g: \Gamma \rightarrow R$. With respect to the usual point multiplication $(f g)(x)=f(x) g(x)$, where $x=\left(x^{1}, x^{2}, \ldots x^{2 n}\right)$ is a set of coordinates on (an 
open subspace of) $\Gamma$, the observables build a commutative algebra. The symplectic structure $\alpha$ allows us to introduce the Poisson bracket of observables as $\{f, g\}(x)=\alpha^{a b} \partial_{a} f(x) \partial_{b} g(x)$, where $\partial_{a}(a=1,2, \ldots 2 n)$ is the (covariant) derivative in $\Gamma$. With respect to the Poisson bracket the set of observables build a Lie-Poisson algebra. The equations of motion in phase space acquire a particular symmetric form in terms of the Poisson brackets $\dot{x}^{a}=\left\{x^{a}, \mathcal{H}\right\}$, a relationship which is valid for any function of phase space coordinates.

In the canonical approach to quantization one replaces the observables by (self-adjoint) operators which act on the Hilbert space. The physical states are vectors in the Hilbert space. Poisson brackets are replaced by commutators which, when applied to the operators associated with the basic observables in phase space, satisfy the canonical commutation relations. Despite its great success especially in the perturbative approach to the physics of elementary particles, this procedure is still not completely understood. The passage from functions to operators is one important step in the canonical approach and despite many efforts done to explain it, today the best way to avoid all kind of existence proofs and mathematical difficulties is just to assume it as a postulate.

Deformation quantization is essentially an attempt to avoid the passage from functions to operators. In fact, it focuses on the algebra of observables of the phase space and replaces the usual point product of functions by a star-product. The canonical commutation relations are now a consequence of the definition of the star-product. An important advantage of this procedure is that quantum as well classical observables are functions defined on the phase space and no operators are required. From the mathematical point of view, the deformation quantization of a given classical system consists in giving an appropriate definition of the star-product which acts (on functions defined) on the phase space. In physics, however, to understand a quantum system one needs to know its quantum states and their energy spectrum. To this end, deformation quantization postulates the existence of a time-evolution function, $\operatorname{Exp}(H t)$, which satisfies the differential equation [13]

$$
i \hbar \frac{d}{d t} \operatorname{Exp}(H t)=H * \operatorname{Exp}(H t)
$$

where $H$ is the Hamiltonian of the classical system. Moreover, it is assumed that the timeevolution function allows a Fourier-Dirichlet expansion as

$$
\operatorname{Exp}(H t)=\sum_{E} \pi_{E} e^{-i t E / \hbar}
$$


where $E$ is the energy (a real number) associated with the state $\pi_{E}$ (distribution on the phase space), or Wigner function, which satisfies the so called *-genvalue equation

$$
H * \pi_{E}=E \pi_{E} .
$$

The states are idempotent and complete. i.e.:

$$
\pi_{E} * \pi_{E^{\prime}}=\delta_{E, E^{\prime}} \pi_{E}, \quad \sum_{E} \pi_{E}=1
$$

As a consequence, the spectral decomposition of the Hamiltonian is give as

$$
H=\sum_{E} E \pi_{E}
$$

Essentially, the objects that are necessary for carrying out the deformation quantization of a physical system are the classical Hamiltonian $H$ and the ${ }^{*}$-product. For a given phase space it is not clear a priori if a consistent ${ }^{*}$-product exists or not and, for a general phase space, this is still an open problem [4]. In the case of free (non-interacting) fields that can be considered heuristically as the sum of an infinite number of harmonic oscillators, it has been shown 7] that the normal star-product is the only admissible star-product. The normal *-product between two functions $f$ and $g$ on phase space is defined by

$$
f *_{N} g=\left.e^{N_{12}} f\left(a^{(1)}, \bar{a}^{(1)}\right) g\left(a^{(2)}, \bar{a}^{(2)}\right)\right|_{a^{(1)}=a^{(2)}=a}, \quad N_{12}=\hbar \delta_{i j} \frac{\partial}{\partial a_{i}^{(1)}} \frac{\partial}{\partial \bar{a}_{j}^{(2)}},
$$

where the superscritpts (1) and (2) denote two arbitrary points in phase space and $a=$ $\left(a_{1}, a_{2}, \ldots, a_{n}\right)$. Furthermore, an overline denotes complex conjugation. The set of phase space variables has to satisfy the standard commutation relations with respect to the Poisson bracket, i.e. $\left\{a_{i}, \bar{a}_{j}\right\}=\delta_{i j},\left\{a_{i}, a_{j}\right\}=\left\{\bar{a}_{i}, \bar{a}_{j}\right\}=0$. In particular one can choose $a_{j}=$ $1 / \sqrt{2}\left(x_{j}+i x_{n+j}\right),(j=1, \ldots, n)$, where we are assuming that the configurational variable $x_{j}$ and its conjugate momentum $x_{n+j}$ have been normalized to come out with the same units.

Let us now apply this procedure to the linearized theory. According to the results given in section [II the canonical variables in the phase space of linearized gravity are the potentials $A_{\alpha}=-(1 / 4) \bar{h}_{\alpha \beta} U^{\beta}$ and their canonical momenta $\Pi_{\alpha}=\dot{A}_{\alpha}$. The Hamiltonian is given by

$$
H=\frac{1}{2} \int d^{3} x\left(\Pi_{\alpha} \Pi^{\alpha}+A_{\alpha, i} A^{\alpha, i}\right) .
$$

The main step in the quantization procedure consists in solving Eq.(3.1) by using the normal *-product (3.6). To this end it is convenient to change from the variables of phase space 
$\left(A_{\alpha}, \Pi_{\alpha}\right)$ to a new set of canonically conjugate variables in which the Hamiltonian takes the simplest possible form. This procedure is very well known in field theory and consists in introducing the momentum representation of the phase variable $A_{\alpha}$ according to [21]

$$
A_{\alpha}(x)=\frac{1}{(2 \pi)^{3 / 2}} \int \frac{d \mathbf{k}}{\sqrt{2 k_{0}}}\left[a_{\alpha}(\mathbf{k}) e^{i k x}+\bar{a}_{\alpha}(\mathbf{k}) e^{-i k x}\right],
$$

where $k$ is a null vector $k_{\mu} k^{\mu}=-k_{0}^{2}+\mathbf{k}^{2}=0$, and $k x=k_{\mu} x^{\mu}$. Then

$$
\Pi_{\alpha}=\dot{A}_{\alpha}=\frac{i}{(2 \pi)^{3 / 2}} \int d \mathbf{k} \sqrt{\frac{k_{0}}{2}}\left[a_{\alpha}(\mathbf{k}) e^{i k x}-\bar{a}_{\alpha}(\mathbf{k}) e^{-i k x}\right],
$$

and

$$
A_{\alpha, j}=\frac{i}{(2 \pi)^{3 / 2}} \int \frac{d \mathbf{k}}{\sqrt{2 k_{0}}} k_{j}\left[a_{\alpha}(\mathbf{k}) e^{i k x}-\bar{a}_{\alpha}(\mathbf{k}) e^{-i k x}\right] .
$$

From the commutation relations for $A_{\alpha}$ and $\Pi_{\beta}$ it can be shown that

$$
\left\{a_{\alpha}, \bar{a}_{\beta}\right\}=\delta_{\alpha \beta}, \quad\left\{a_{\alpha}, a_{\beta}\right\}=\left\{\bar{a}_{\alpha}, \bar{a}_{\beta}\right\}=0,
$$

where the same value of $\mathbf{k}$ has been assumed in all the arguments. Introducing (3.9) and (3.10) into the Hamiltonian (3.7) and performing some of the integrations we obtain

$$
H=\frac{1}{2} \int k_{0} \eta^{\alpha \beta} a_{\alpha}(\mathbf{k}) \bar{a}_{\beta}(\mathbf{k}) d \mathbf{k}
$$

We see that the resulting Hamiltonian is linear in the new variables and does not contain derivatives. It can be interpreted as an infinite sum of harmonic oscillators. This is an important observation [7, 12, 15] that allows us to formally apply the normal *-product as defined in (3.6). In fact, when going from a system with a finite number of degrees of freedom to a field theory, one only has to "replace" partial derivatives by variational derivatives. We use this fact to calculate time-evolution function as the solution of Eq.(3.1). Then we have

$$
\ln \operatorname{Exp}_{N}(H t)=\frac{1}{\hbar}\left(e^{-i k_{0} t}-1\right) \int \eta^{\alpha \beta} a_{\alpha}(\mathbf{k}) \bar{a}_{\beta}(\mathbf{k}) d \mathbf{k},
$$

where the subscript $N$ indicates that in Eq. (3.1) the normal *-product has been used. Using the definition of the exponential of a functional, the last expression can be written as

$$
\operatorname{Exp}_{N}(H t)=\exp \left(-\frac{1}{\hbar} \int \eta^{\alpha \beta} a_{\alpha}(\mathbf{k}) \bar{a}_{\beta}(\mathbf{k}) d \mathbf{k}\right) \sum_{n=0}^{\infty} \frac{e^{-i n k_{0} t}}{n ! \hbar^{n}} \int \eta^{\alpha \beta} a_{\alpha}^{n}(\mathbf{k}) \bar{a}_{\beta}^{n}(\mathbf{k}) d \mathbf{k} .
$$

Comparing this expression with the Fourier-Dirichlet expansion (3.2) we can identify the corresponding states as

$$
\pi_{E_{0}}^{N}=\exp \left(-\frac{1}{\hbar} \int \eta^{\alpha \beta} a_{\alpha}(\mathbf{k}) \bar{a}_{\beta}(\mathbf{k}) d \mathbf{k}\right)
$$




$$
\pi_{E_{n}}^{N}=\frac{1}{n ! \hbar^{n}} \pi_{E_{0}}^{N} \int \eta^{\alpha \beta} a_{\alpha}^{n}(\mathbf{k}) \bar{a}_{\beta}^{n}(\mathbf{k}) d \mathbf{k}
$$

and the energy spectrum

$$
E_{n}=n \hbar k_{0}
$$

In this manner we have arrived at the main result of quantization: The determination of the quantum states and the energy spectrum of the system. The main advantage of deformation quantization consists in achieving this goal without using the operator formalism. Now we are confronted with the problem of finding the physical significance of our results. To this end, let us remember that at the classical level we have derived Einsteins linearized equations directly from the Lagrangian (2.12), and have noticed that the Lorentz gauge conditions have to be postulated as an additional requirement. It seems therefore natural to impose this requirement on the quantum states to find out which of them are physical. From Eq.(3.8) we find that the Lorentz gauge conditions are equivalent to

$$
A_{\alpha}^{, \alpha}=\frac{1}{(2 \pi)^{3 / 2}} \int \frac{d \mathbf{k}}{\sqrt{2 k_{0}}} i k^{\alpha}\left[a_{\alpha}(\mathbf{k}) e^{i k x}-\bar{a}_{\alpha}(\mathbf{k}) e^{-i k x}\right]=0 .
$$

Clearly, this condition is identically satisfied if

$$
k^{\alpha} a_{\alpha}(\mathbf{k})=0,
$$

what implies that only three components of $a_{\alpha}(\mathbf{k})$ are linearly independent. Furthermore, the gauge freedom given by Eq.(2.15) implies that the harmonic function $\Sigma(x)$ can be used to eliminate an additional component of $a_{\alpha}(\mathbf{k})$. So we are left with only two true components, say, $a_{1}(\mathbf{k})$ and $a_{2}(\mathbf{k})$. This is in accordance with the fact that gravitational fields possess only two physical degrees of freedom [18]. From Eqs.(3.15) and (3.16) we see that all states are specified as powers of $a_{1}(\mathbf{k})$ and $a_{2}(\mathbf{k})$. The results should not depend on the choice of these two linear independent components, but one can use these freedom to adapt the formalism to different physical situations. For instance, in the case of the Newtonian limit it seems reasonable to choose the Newtonian potential $\phi$ and one of the "gravitomagnetic" functions [19], say $\gamma$, as independent configuration variables so that $A_{\alpha}=-(1 / 4)(4 \phi, \gamma, 0,0)$. In the case of gravitational waves a more suitable choice would be $A_{\alpha}=-(1 / 4)\left(0, \gamma_{1}, \gamma_{2}, 0\right)$ where $\gamma_{1}$ and $\gamma_{2}$ are now related to the special combination of the metric components that describe gravitational waves (see, for instance, [19]). 
Independently of the choice of gauge, the states and spectrum are represented by Eqs.(3.15), (3.16) and (3.17). The coefficients $a_{\alpha}(\mathbf{k})$ can be interpreted as densities that determine distributions in phase space, i.e. as state densities. But the formalism of deformation quantization allows a transition to the operator formalism according to certain fixed rules [13]. In that case, one would expect that the operator counterparts of $a_{\alpha}$ and $\bar{a}_{\alpha}$ would correspond to the annihilation and creation operators of standard quantum field theory. The energy spectrum (3.17) is discrete with vanishing zero-point energy. If we would use the Moyal product for the quantization, we would obtain a non-vanishing zero point energy and would be confronted with the problem of divergencies that commonly appears in perturbative quantum field theory [7].

\section{CONCLUSIONS}

The aim of this work was to apply the formalism of deformation quantization to linearized Einstein's equations. We first show two alternative ways to consider Einstein's linearized equations in a field theoretical approach. We then use the modified Maxwell representation of linearized gravity to calculate the classical Hamiltonian of the theory, avoiding the problem of a singular Lagrangian. The Hamiltonian is one of the main ingredients necessary to carry out the deformation quantization of any physical system. We use the normal star-product to derive the commutation relations, in analogy with other free (linear) fields. The expression for the time-evolution function is found explicitly, and the Fourier-Dirichlet expansion of the Hamiltonian is used to derive the energy spectrum and the complete set of states of the system. A more detailed analysis is necessary in order to clarify further the physical meaning of the states. We have used the momentum representation in analogy with the standard methods of quantum field theory. For this reason, the results of the quantization are more

adapted to a possible interpretation in terms of elementary particles and not in terms of a possible quantization of space and time. This, however, is a much more complicated problem that requires a separate and detailed treatment. 


\section{Acknowledgments}

It is a great pleasure to dedicate this work to Alberto García on his 60-th birthday. This work was in part supported by DGAPA-UNAM grant IN112401, CONACyT grant 36581-E, and US DOE grant DE-FG03-91ER40674. H.Q. thanks UC-MEXUS for support.

[1] H. J. Groenewold, Physica (Amsterdam) 12, 405 (1946); L. van Hove, Proc. R. Acad. Sci. Belgium 26, 1 (1951).

[2] A. Weinstein, Semin. Bourbaki, Asterique 789, 389 (1995).

[3] C. Zachos, hep-th/0110114

[4] S. Waldmann, hep-th/0303080

[5] A. C. Hirshfeld and P. Henselder, Am. J. Phys. 70, 537 (2002).

[6] F. Bayen, M. Flato, C. Fronsdal, A. Lichnerowicz, and D. Sternheimer, Ann. Phys. (N.Y.) 111, 61 (1978).

[7] J. Dito, Lett. Math. Phys. 20, 125 (1990).

[8] M. Kontsevich, q-alg/9709040.

[9] M. Dütch and K. Fredenhagen, hep-th/9807215, hep-th/0101079.

[10] M. Bordemann, H.C. Herbig and S. Waldmann, Comm. Math. Phys. 210, 107 (2000).

[11] A. S. Cattaneo and G. Felder, Comm. Math. Phys. 212, 591 (2000).

[12] H. Garcia-Compean, J.F. Plebanski, M. Przanowski and F.J. Turrubiates, Int. J. Mod. Phys. A 16, 2533 (2001).

[13] A. C. Hirshfeld and P. Henselder, Ann. Phys. 298, 382 (2002).

[14] F. Antonsen, gr-qc/9712012.

[15] H. Garcia-Compean, J.F. Plebanski, M. Przanowski and F.J. Turrubiates, J. Phys. A 33, 7935 (2000).

[16] D. Minic, hep-th/9909022.

[17] F. Antonsen, gr-qc/9710021.

[18] R. M. Wald, General Relativity (The University of Chicago Press, Chicago, 1984).

[19] C. W. Misner, K. S. Thorne, and J. A. Wheeler, Gravitation (Freeman Ed., San Francisco, 1973). 
[20] D. M. Gitman and I. V. Tyutin, Quantization of fields with constraints (Springer-Verlag, Berlin, 1990).

[21] N. N. Bogoliubov and D. V. Shirkov, Quantum fields (Benjamin Ed., USA, 1983) 